\title{
A new error estimate on uniform norm of a parabolic variational inequality with nonlinear source terms via the subsolution concepts
}

Salah Boulaaras ${ }^{1,2^{*}}$, Mohamed El Amine Bencheikh Le Hocine ${ }^{3,4}$ and Mohamed Haiour ${ }^{4}$

This presented work is in memory of the first author's father (1910-1999) Mr. Mahmoud ben Mouha Boulaaras

${ }^{*}$ Correspondence:

saleh_boulaares@yahoo.fr;

S.Boularas@qu.edu.sa

${ }^{1}$ Department of Mathematics,

College of Sciences and Arts, Al-Ras

Qassim University, Buraydah,

Kingdom of Saudi Arabia

${ }^{2}$ Laboratory of Fundamental and

Applied Mathematics of Oran

(LMFAO), University of Oran 1,

Ahmed Benbella, Algeria

Full list of author information is

available at the end of the article

\section{黛 Springer}

\begin{abstract}
This paper deals with the numerical analysis of parabolic variational inequalities with nonlinear source terms, where the existence and uniqueness of the solution is provided by using Banach's fixed point theorem. In addition, an optimally $L^{\infty}$-asymptotic behavior is proved using Euler time scheme combined with the finite element spatial approximation. The approach is based on Bensoussan-Lions algorithm for evolutionary free boundary problems using the concepts of subsolutions.
\end{abstract}

MSC: $65 \mathrm{~N} 30 ; 65 \mathrm{~K} 15 ; 05 \mathrm{C} 38 ; 65 \mathrm{~N} 15$

Keywords: PVIs; Nonlinear source terms; Subsolution concepts; Existence and uniqueness; Bensoussan-Lions algorithm

\section{Introduction}

We consider the following parabolic variational inequality: Find $u \in L^{2}\left(0, T ; H_{0}^{1}(\Omega)\right)$ such that

$$
\left\{\begin{array}{l}
\frac{\partial u}{\partial t}+\mathcal{L} u-f(u) \leq 0 ; \quad u \leq \psi \\
\left.\left(\frac{\partial u}{\partial t}+\mathcal{L} u-f(u)\right)(u-\psi)=0 \quad \text { in } Q_{T}:=\right] 0, T[\times \Omega \\
\left.u=0 \text { on } \sum_{T}:=\right] 0, T[\times \partial \Omega \\
u(0, \cdot)=u_{0}, \quad \text { on } \Omega
\end{array}\right.
$$

where $\Omega$ is a bounded smooth and regular domain of $\mathbb{R}^{d}, d \geq 1$, with smooth boundary $\partial \Omega$; the $f(\cdot)$ and $u_{0}=u_{0}(x)$ are given data; the $\psi$ is a regular function in $L^{2}\left(0, T ; W^{2, \infty}(\Omega)\right)$, and the $\mathcal{L}$ is a second-order, uniformly elliptic operator of the form

$$
\mathcal{L}=-\sum_{j, k=1}^{d} a_{j k}(x) \frac{\partial^{2}}{\partial x_{j} \partial x_{k}}+\sum_{j=1}^{d} b_{j}(x) \frac{\partial}{\partial x_{j}}+a_{0}(x),
$$

(c) The Author(s) 2020. This article is licensed under a Creative Commons Attribution 4.0 International License, which permits use sharing, adaptation, distribution and reproduction in any medium or format, as long as you give appropriate credit to the original author(s) and the source, provide a link to the Creative Commons licence, and indicate if changes were made. The images or other third party material in this article are included in the article's Creative Commons licence, unless indicated otherwise in a credit line to the material. If material is not included in the article's Creative Commons licence and your intended use is not permitted by statutory regulation or exceeds the permitted use, you will need to obtain permission directly from the copyright holder. To view a copy of this licence, visit http://creativecommons.org/licenses/by/4.0/. 
Parabolic variational inequality (1.1) has arisen from many scientific, engineering, and economic problems such as heat control problem, Stefan problem, and American option problem (see [3, 5-13, 16, 18, 19, 21]).

In this paper, we give an $L^{\infty}$-error estimate for the numerical approximation of the solution of problem (1.1). From [2] (see also [8]), we know that (1.1) can be approximated by the following parabolic variational inequality with nonlinear source terms (PVI): Find $u(x, t)$ such that $u \in L^{2}\left(0, T ; H_{0}^{1}(\Omega)\right), \frac{\partial u}{\partial t} \in L^{2}\left(0, T ; L^{2}(\Omega)\right)$, and

$$
\left\{\begin{array}{l}
\left(\frac{\partial u}{\partial t}, v-u\right)+a(u, v-u) \geq(f(u), v-u), \quad \text { for all } v \in H_{0}^{1}(\Omega), t \in(0, T] \\
u \leq \psi, \quad v \leq \psi \\
u(x, 0)=u_{0}(x) \text { in } \Omega
\end{array}\right.
$$

where $a(\cdot, \cdot)$ is a bilinear form continuous on $H^{1}(\Omega) \times H^{1}(\Omega)$ corresponding to elliptic operator $\mathcal{L}$ of second order defined as follows:

$$
a(u, v)=\int_{\Omega}\left(\sum_{j, k=1}^{d} a_{j k}(x) \frac{\partial u}{\partial x_{j}} \frac{\partial v}{\partial x_{k}}+\sum_{k=1}^{d} b_{k}(x) \frac{\partial u}{\partial x_{k}} v+a_{0}(x) u v\right) d x
$$

with $a_{j k}(\cdot), b_{j}(\cdot), a_{0}(\cdot)$, smooth coefficients satisfying the following conditions:

$$
\left\{\begin{array}{l}
a_{j k}(x)=a_{k j}(x), \\
a_{0}(x) \geq \beta>0, \quad \beta \text { is a constant }
\end{array}\right.
$$

and for each $\xi \in \mathbb{R}^{d}$ and for almost every $x \in \Omega$,

$$
\sum_{j, k=1}^{d} a_{j k}(x) \xi_{j} \xi_{k} \geq \alpha_{0}|\xi|^{2} \quad \text { with constant } \alpha_{0}>0
$$

According to Theorem 2.3 in [8], there exists $\gamma>0$ such that

$$
a(\varphi, \varphi)+\lambda\|\varphi\|_{L^{2}(\Omega)}^{2} \geq \gamma\|\varphi\|_{H_{0}^{1}(\Omega)}^{2}, \quad \forall \varphi \in H_{0}^{1}(\Omega) \text {, with } \gamma>0 .
$$

The function $f(\cdot)$ is a nondecreasing and Lipschitz continuous nonlinearity

$$
f \in L^{2}\left(0, T ; L^{\infty}(\Omega)\right) \cap C^{1}\left(0, T ; H^{-1}(\Omega)\right), \quad f \geq 0,
$$

with Lipschitz constant $\alpha>0$, satisfying the following assumption:

$$
\alpha<\beta,
$$

where $\beta$ is the constant defined in (1.3). The symbol $(\cdot, \cdot)$ is the inner product in $L^{2}(\Omega)$.

Error estimates for piecewise linear finite element approximations of parabolic variational inequalities with linear source terms have been established in various papers: in [20] and [9] an $L^{2}$-error estimate is given by using a backward differencing in time. Also an $L^{2}$-error estimate is given in [23] by using a general finite difference discretization in 
time. Reference [4] gives an $L^{2}$-error estimate using the discretized truncation method. In [1] and [22] a posteriori error estimates have been proved. An $L^{\infty}$-error estimate has been proved in [15] and [17]. Recently an $L^{\infty}$-asymptotic behavior has been considered in [2] by using a semi-implicit time scheme combined with the finite element spatial approximation.

In this paper, we introduce a new approach to derive optimal $L^{\infty}$-asymptotic behavior for parabolic variational inequality with nonlinear source terms. This approach is based on Bensoussan-Lions algorithm for evolutionary free boundary problems using the concepts of subsolutions.

The paper is organized as follows. In Sect. 2, we state the continuous problem and study some qualitative properties. In Sect. 3, we consider the discrete problem and set up analogous discrete qualitative properties. In Sect. 4 , we derive an $L^{\infty}$-error estimate of the approximation and we give the main result of the paper.

\section{Semi continuous problem}

\subsection{Time discretization}

In order to obtain a full discretization of (1.3), we consider a uniform mesh for the time variable $t$ and define

$$
t_{n}=n \Delta t, \quad n=0,1, \ldots, \mathcal{N},
$$

$\Delta t>0$ being the time-step, and $\mathcal{N}=\left[\frac{T}{\Delta t}\right]$, the integral part of $\frac{T}{\Delta t}$.

Next, we replace the time derivative by means of suitable difference quotients, thus constructing a sequence $u^{n}(x) \in H_{0}^{1}(\Omega)$ that approaches $u\left(t_{n}, x\right)$. For simplicity, we confine ourselves to the so-called semi-implicit scheme, which consists in replacing (1.3) by the following scheme: Find $u^{n} \in H_{0}^{1}(\Omega)$ such that

$$
\left\{\begin{array}{l}
\left(\frac{1}{\Delta t}\left(u^{n}-u^{n-1}\right), v-u^{n}\right)+a\left(u^{n}, v-u^{n}\right) \geq\left(f\left(u^{n}\right), v-u^{n}\right), \quad v \in H_{0}^{1}(\Omega) \\
u^{n} \leq \psi, \quad v \leq \psi, \quad n=1, \ldots, \mathcal{N}
\end{array}\right.
$$

where

$$
\Delta t=\frac{T}{\mathcal{N}} .
$$

By adding $\left(\frac{u^{n-1}}{\Delta t}, v-u^{n}\right)$ to both parties of inequalities (2.2), we get

$$
\left\{\begin{array}{l}
\frac{1}{\Delta t}\left(u^{n}, v-u^{n}\right)+a\left(u^{n}, v-u^{n}\right) \geq\left(f\left(u^{n}\right)+\frac{1}{\Delta t} u^{n-1}, v-u^{n}\right) \\
u^{n} \leq \psi, \quad v \leq \psi, \quad n=0,1, \ldots, \mathcal{N}
\end{array}\right.
$$

As the bilinear form $a(\cdot, \cdot)$ is noncoercive in $H_{0}^{1}(\Omega)$.

Set

$$
b(u, v)=a(u, v)+\lambda(u, v) .
$$


Then the bilinear form $b(u, v)$ is an elliptic, and therefore (2.4) can be written as the following coercive elliptic variational inequalities: Find $u^{n} \in H_{0}^{1}(\Omega)$ such that

$$
\left\{\begin{array}{l}
b\left(u^{n}, v-u^{n}\right) \geq\left(f\left(u^{n}\right)+\lambda u^{n-1}, v-u^{n}\right), \quad v \in H_{0}^{1}(\Omega), \\
u^{n} \leq \psi, \quad v \leq \psi, \quad n=0,1, \ldots, \mathcal{N},
\end{array}\right.
$$

where

$$
\left\{\begin{array}{l}
b\left(u^{n}, v-u^{n}\right)=a\left(u^{n}, v-u^{n}\right)+\lambda\left(u^{n}, v-u^{n}\right), \quad v \in H_{0}^{1}(\Omega), \\
\lambda=\frac{1}{\Delta t}>0 .
\end{array}\right.
$$

Remark 1 Equation (2.6) is called the coercive continuous problem of elliptic variational inequalities (VI).

Notation 1 We denote by $u^{n}=\partial\left(f\left(u^{n}\right), \psi\right)$ the solution of problem (2.6).

\subsection{Existence and uniqueness}

Next, using the preceding assumptions, we prove the existence of a unique solution for problem (2.6) by means of Banach's fixed point theorem.

\subsubsection{A fixed point mapping associated with continuous problem (2.6)}

We consider the following mapping:

$$
\begin{gathered}
\mathbb{T}: L^{\infty}(\Omega) \longrightarrow L^{\infty}(\Omega), \\
w \rightarrow \mathbb{T} w=\zeta^{n},
\end{gathered}
$$

where $\xi^{n}=\sigma(f(w), \psi)$ is the solution to the following variational inequalities:

$$
\left\{\begin{array}{l}
b\left(\zeta^{n}, v-\zeta^{n}\right) \geq\left(f(w)+\lambda w, v-\zeta^{n}\right), \quad v \in H_{0}^{1}(\Omega) \\
\zeta^{n} \leq \psi, \quad v \leq \psi
\end{array}\right.
$$

Problem (2.10) being a coercive VI, thanks to [3] and [10], has one and only one solution.

Theorem 1 Under the preceding hypotheses and notation, the mapping $\mathbb{T}$ is a contraction in $L^{\infty}(\Omega)$ with a contraction constant $\left(\frac{\alpha \Delta t+1}{\beta \Delta t+1}\right)$. Therefore, $\mathbb{T}$ admits a unique fixed point which coincides with the solution of problem (2.6).

Proof In [13], by taking $\lambda=\frac{1}{\Delta t}$, we can easily get

$$
\|\mathbb{T} w-\mathbb{T} \tilde{w}\|_{\infty} \leq\left(\frac{\alpha \Delta t+1}{\beta \Delta t+1}\right)\|w-\tilde{w}\|_{\infty} .
$$

The mapping $\mathbb{T}$ clearly generates the following continuous algorithm. 


\subsection{A continuous iterative scheme}

A continuous iterative scheme for the solution of problem (2.6) is given as follows.

Starting from $u^{0}=u_{0}$ the solution of the following equation:

$$
b\left(u_{0}, v\right)=\left(f\left(u_{0}\right)+\lambda u_{0}, v\right), \quad \forall v \in H_{0}^{1}(\Omega) .
$$

Now, we give the following algorithm:

$$
u^{n}=\mathbb{T} u^{n-1}, \quad n=1, \ldots, \mathcal{N}-1,
$$

where $u^{n}$ is the solution to (2.6).

Making use of the propriety of mapping $\mathbb{T}$, we have the following geometric convergence result.

Proposition 1 Let $\rho=\frac{\alpha \Delta t+1}{\beta \Delta t+1}$, under conditions of Theorem 1, we have

$$
\left\|u^{n}-u^{\infty}\right\|_{\infty} \leq \rho^{n}\left\|u_{0}-u^{\infty}\right\|_{\infty}
$$

where $u^{\infty}$ is the asymptotic solution of the problem of variational inequalities: Find $u^{\infty} \in$ $H_{0}^{1}(\Omega)$ such that

$$
\left\{\begin{array}{l}
b\left(u^{\infty}, v-u^{\infty}\right) \geq\left(f\left(u^{\infty}\right)+\lambda u^{\infty}, v-u^{\infty}\right), \quad v \in H_{0}^{1}(\Omega), \\
u^{\infty} \leq \psi, \quad v \leq \psi
\end{array}\right.
$$

Proof We adapt [2].

In what follows, we give some qualitative properties of the solution of problem (2.6).

\subsection{Some qualitative properties of the solution of (2.6)}

The solution $u^{n}$ of (2.6) possesses the following properties.

2.4.1 A monotonicity property

Let $u^{n}=\partial\left(F\left(u^{n}\right), \psi\right)\left(\right.$ resp. $\left.\tilde{u}^{n}=\partial\left(\tilde{F}\left(\tilde{u}^{n}\right), \tilde{\psi}\right)\right)$ be the solution of problem (2.6) with righthand side $F\left(u^{n}\right)=f\left(u^{n}\right)+\lambda u^{n-1}\left(\operatorname{resp} . \tilde{F}\left(\tilde{u}^{n}\right)=\tilde{f}\left(\tilde{u}^{n}\right)+\lambda \tilde{u}^{n-1}\right)$. Then we have the following.

Lemma 1 (cf. [6] and [10]) If $F\left(u^{n}\right) \geq \tilde{F}\left(\tilde{u}^{n}\right)$ and $\psi \geq \tilde{\psi}$, then

$$
\partial\left(F\left(u^{n}\right), \psi\right) \geq \partial\left(\tilde{F}\left(\tilde{u}^{n}\right), \tilde{\psi}\right) .
$$

2.4.2 A continuous $L^{\infty}$-stability property

Proposition 2 Under conditions of Lemma 1, we have

$$
\left\|\partial\left(F\left(u^{n}\right), \psi\right)-\partial\left(\tilde{F}\left(\tilde{u}^{n}\right), \tilde{\psi}\right)\right\|_{\infty} \leq \frac{1}{\beta}\left\|f\left(u^{n}\right)-\tilde{f}\left(\tilde{u}^{n}\right)\right\|_{\infty} .
$$

Proof Let

$$
\phi=\frac{1}{\lambda+\beta}\left\|f\left(u^{n}\right)-\tilde{f}\left(\tilde{u}^{n}\right)\right\|_{\infty} .
$$


Then, from (1.5), it is easy to see that

$$
\tilde{F}\left(\tilde{u}^{n}\right) \leq F\left(u^{n}\right)+\frac{\lambda+a_{0}}{\lambda+\beta}\left\|f\left(u^{n}\right)-\tilde{f}\left(\tilde{u}^{n}\right)\right\|_{\infty}=F\left(u^{n}\right)+\left(\lambda+a_{0}\right) \cdot \phi .
$$

So, due to Lemma 1, it follows that

$$
\partial\left(\tilde{F}\left(\tilde{u}^{n}\right), \tilde{\psi}\right) \leq \partial\left(F\left(u^{n}\right)+\left(\lambda+a_{0}\right) \cdot \phi, \psi+\phi\right) \leq \partial\left(F\left(u^{n}\right), \psi\right)+\phi,
$$

hence

$$
\partial\left(\tilde{F}\left(\tilde{u}^{n}\right), \tilde{\psi}\right)-\partial\left(F\left(u^{n}\right), \psi\right) \leq \phi
$$

Interchanging the role of $F\left(u^{n}\right)$ and $\tilde{F}^{n}$, we also get

$$
\partial\left(F\left(u^{n}\right), \psi\right)-\partial\left(\tilde{F}^{n}, \tilde{\psi}\right) \leq \phi
$$

Then, from (2.8), it is easy to see that

$$
\begin{aligned}
\left\|\partial\left(F\left(u^{n}\right), \psi\right)-\partial\left(\tilde{F}\left(\tilde{u}^{n}\right), \tilde{\psi}\right)\right\|_{\infty} & \leq \frac{\Delta t}{\beta \Delta t+1}\left\|f\left(u^{n}\right)-\tilde{f}\left(\tilde{u}^{n}\right)\right\|_{\infty} \\
& \leq \frac{1}{\beta\left(1+\frac{1}{\beta \Delta t}\right)}\left\|f\left(u^{n}\right)-\tilde{f}\left(\tilde{u}^{n}\right)\right\|_{\infty} \\
& \leq \frac{1}{\beta}\left\|f\left(u^{n}\right)-\tilde{f}\left(\tilde{u}^{n}\right)\right\|_{\infty}
\end{aligned}
$$

which completes the proof.

\subsubsection{The concept of continuous subsolution property}

Definition $1 z^{n} \in H_{0}^{1}(\Omega)$ is said to be a continuous subsolution for the problem of VI (2.6) if

$$
\left\{\begin{array}{l}
b\left(z^{n}, v\right) \leq\left(f\left(z^{n}\right)+\lambda z^{n}, v\right), \quad v \in H_{0}^{1}(\Omega), \\
z^{n} \leq \psi, \quad v \geq 0, \quad n=1, \ldots, \mathcal{N}-1 .
\end{array}\right.
$$

Theorem 2 (cf. [6]) Let $\mathbb{X}$ denote the set of such subsolutions, then the solution of (2.6) is the least upper bound of $\mathbb{X}$.

\section{The discrete problem}

Let $\Omega$ be decomposed into triangles, and let $\tau_{h}$ denote the set of all those elements; $h>0$ is the mesh size. We assume that the family $\tau_{h}$ is regular and quasi-uniform. We consider $\phi_{l}, l=1,2, \ldots, m(h)$, the usual basis of affine functions defined by $\phi_{l}\left(M_{s}\right)=\delta_{l, s}$, where $M_{s}$ is a vertex of the considered triangulation.

Let us $\mathbb{V}_{h}$ denote the standard piecewise linear finite element space such that

$$
\mathbb{V}_{h}=\left\{\begin{array}{l}
v_{h} \in C^{0}(\bar{\Omega}), v_{h}=0 \text { on } \partial \Omega \text { such that: } \\
\left.v_{h}\right|_{K^{i}} \in P_{1}, K \in \tau_{h}, v_{h} \leq r_{h} \psi, v_{h}(\cdot, 0)=v_{0 h} \text { in } \Omega
\end{array}\right\} .
$$


The interpolation operator is applied to the function $v$ continuous, defined by

$$
r_{h} v=\sum_{l=1}^{m(h)} v\left(M_{l}\right) \phi_{l}(x)
$$

and $\mathbb{B}$ is the matrix with generic entries

$$
(\mathbb{B})_{l, s}=a\left(\phi_{l}, \phi_{s}\right), \quad 1 \leq l, s \leq m(h) .
$$

In the sequel of the paper, we use the discrete maximum assumption (d.m.p.). In other words, we assume that the matrix $\mathbb{B}$ is an M-matrix (cf. [14]).

Remark 2 Under the d.m.p., we achieve a similar study to that devoted to the continuous problem; therefore the qualitative properties and results stated in the continuous case are conserved in the discrete case.

As in the continuous situation, one can tackle the discrete problem by considering the equivalent formulation: Find $u_{h}^{n} \in \mathbb{V}_{h}$ such that

$$
\left\{\begin{array}{l}
b\left(u_{h}^{n}, v_{h}-u_{h}^{n}\right) \geq\left(f\left(u_{h}^{n}\right)+\lambda u_{h}^{n}, v_{h}-u_{h}^{n}\right), \quad v_{h} \in \mathbb{V}_{h}, \\
u_{h}^{n} \leq r_{h} \psi, \quad v_{h} \leq r_{h} \psi .
\end{array}\right.
$$

Notation 2 We denote by $u_{h}^{n}=\partial_{h}\left(f^{n}\left(u_{h}^{n}\right), r_{h} \psi\right)$ the solution of problem (3.4).

Existence and uniqueness of a solution of problem (3.4) can be shown similarly to that of the continuous case provided the discrete maximum principle is satisfied.

\subsection{Existence and uniqueness}

3.1.1 A fixed point mapping associated with discrete problem (3.4)

We consider the following mapping:

$$
\begin{array}{r}
\mathbb{T}: L^{\infty}(\Omega) \longrightarrow \mathbb{V}_{h}, \\
w \rightarrow \mathbb{T}_{h} w=\xi_{h}^{n},
\end{array}
$$

where $\xi_{h}^{n}=\sigma_{h}\left(f^{n}(w), r_{h} \psi\right)$ is a solution of the following discrete coercive VI:

$$
\left\{\begin{array}{l}
b\left(\xi_{h}^{n}, v-\xi_{h}^{n}\right) \geq\left(f(w)+\lambda w, v-\xi_{h}^{n}\right), \quad v_{h} \in \mathbb{V}_{h}, \\
\xi_{h}^{n} \leq r_{h} \psi, \quad v \leq r_{h} \psi .
\end{array}\right.
$$

Theorem 3 Under the d.m.p. assumption and the preceding hypotheses and notation, the mapping $\mathbb{T}_{h}$ is a contraction in $L^{\infty}(\Omega)$ with a contraction constant $\left(\frac{\alpha \Delta t+1}{\beta \Delta t+1}\right)$. Therefore, $\mathbb{T}_{h}$ admits a unique fixed point which coincides with the solution of problem (3.4).

As in the continuous situation, one can define the following discrete iterative scheme. 


\subsection{A discrete iterative scheme}

A discrete iterative scheme for the solution of problem (3.4) is given as follows.

Starting from $u_{h}^{0}=u_{0, h}$, the solution of the following equation:

$$
b\left(u_{0, h}, v_{h}\right)=\left(f\left(u_{0, h}\right)+\lambda u_{0, h}, v_{h}\right), \quad v_{h} \in \mathbb{V}_{h} .
$$

Now, we give the following algorithm:

$$
u_{h}^{n}=\mathbb{T}_{h} u_{h}^{n-1}, \quad n=1, \ldots, \mathcal{N}-1,
$$

where $u_{h}^{n}$ is a solution of problem (3.4).

Using the above result, we are able to establish the following geometric convergence of sequence $u_{h}^{n}$.

Proposition 3 Let $\rho=\frac{\alpha \Delta t+1}{\beta \Delta t+1}$, under the d.m.p. assumption and Theorem 3, we have

$$
\left\|u_{h}^{n}-u_{h}^{\infty}\right\|_{\infty} \leq \rho^{n}\left\|u_{h, 0}-u_{h}^{\infty}\right\|_{\infty}
$$

where $u_{h}^{\infty}$ is the asymptotic solution of problem of variational inequalities: Find $u_{h}^{\infty} \in \mathbb{V}_{h}$ such that

$$
\left\{\begin{array}{l}
b\left(u_{h}^{\infty}, v-u_{h}^{\infty}\right) \geq\left(f\left(u_{h}^{\infty}\right)+\lambda u_{h}^{\infty}, v_{h}-u_{h}^{\infty}\right), \quad v_{h} \in \mathbb{V}_{h}, \\
u_{h}^{\infty} \leq r_{h} \psi, \quad v_{h} \leq r_{h} \psi .
\end{array}\right.
$$

Proof It is very similar to that of the continuous case.

Under the d.m.p., the solution of discrete problem (3.4) possesses analogous properties to those of the continuous problem.

\subsection{Some qualitative properties of the solution of (3.4)}

As in the continuous situation, the solution $u_{h}^{n}$ of system (3.4) possesses the following properties.

\subsubsection{A monotonicity property}

Let $u_{h}^{n}=\partial_{h}\left(F^{n}, r_{h} \psi\right)\left(\right.$ resp. $\left.\tilde{u}_{h}^{n}=\partial_{h}\left(\tilde{F}^{n}, r_{h} \tilde{\psi}\right)\right)$ the solution to (3.4) with right-hand side $F^{n}$.

Lemma 2 If $F^{n} \geq \tilde{F}^{n}$ and $\psi \geq \tilde{\psi}$, then

$$
\partial_{h}\left(F^{n}, r_{h} \psi\right) \geq \partial_{h}\left(\tilde{F}^{n}, r_{h} \tilde{\psi}\right) .
$$

\subsubsection{A discrete $L^{\infty}$-stability}

Proposition 4 Under the d.m.p. assumption and conditions of Lemma 2, we have

$$
\left\|\partial_{h}\left(F\left(u^{n}\right), r_{h} \psi\right)-\partial_{h}\left(\tilde{F}\left(\tilde{u}^{n}\right), r_{h} \tilde{\psi}\right)\right\|_{\infty} \leq \frac{1}{\beta}\left\|f\left(u_{h}^{n}\right)-\tilde{f}\left(\tilde{u}_{h}^{n}\right)\right\|_{\infty} .
$$

Proof It is very similar to that of the continuous case. 


\subsubsection{The concept of discrete subsolution}

Definition $2 z_{h}^{n} \in \mathbb{V}_{h}$ is said to be a discrete subsolution for the system of quasi-variational inequalities (3.4) if

$$
\left\{\begin{array}{l}
b\left(z_{h}, \varphi_{s}\right) \leq\left(f\left(z_{h}\right)+\lambda z_{h}, \varphi_{s}\right), \quad \forall s=1, \ldots, m(h) \\
z_{h} \leq r_{h} \psi, \quad \varphi_{s} \geq 0 .
\end{array}\right.
$$

Theorem 4 Let $\mathbb{X}_{h}$ be the set of such subsolutions, then under the d.m.p., the solution of (3.4) is the least upper bound of the set $\mathbb{X}_{h}$.

\section{Finite element error analysis}

This section is devoted to deriving an error estimate, in the maximum norm, between the $n$th iterates $u^{n}$ and their finite element counterpart $u_{h}^{n}$. For that we first introduce two auxiliary sequences.

\subsection{Two auxiliary sequences}

\subsubsection{A discrete sequence}

We define the following discrete sequence $\left\{\bar{u}_{h}^{n}\right\}_{n \geq 1}$, where $\bar{u}_{h}^{n}$ is a solution to the following discrete problem of variational inequalities (VI):

$$
\left\{\begin{array}{l}
b\left(\bar{u}_{h}^{n}, v_{h}-\bar{u}_{h}^{n}\right) \geq\left(f\left(u^{n}\right)+\lambda \cdot u^{n}, v_{h}-\bar{u}_{h}^{n}\right), \quad v_{h} \in \mathbb{V}_{h}, \\
\bar{u}_{h}^{n} \leq r_{h} \psi, \quad v_{h} \leq r_{h} \psi,
\end{array}\right.
$$

where $u^{n}$ is the solution to (2.6).

Lemma 3 (cf. [13]) There exists a constant $C$ independent of $h, n$, and $\Delta t$ such that

$$
\left\|\bar{u}_{h}^{n}-u^{n}\right\|_{\infty} \leq C h^{2}|\log h|^{2}
$$

Proposition 5 There exists a sequence of discrete subsolutions $\left\{\alpha_{h}^{n}\right\}_{n \geq 1}$ such that

$$
\left\{\begin{array}{l}
\alpha_{h}^{n} \leq u_{h}^{n}, \\
\text { and } \\
\left\|\alpha_{h}^{n}-u^{n}\right\|_{\infty} \leq C h^{2}|\log h|^{2},
\end{array}\right.
$$

where the constant $C$ is independent of $h, \Delta t$, and $n$.

Proof For $n=1$, we consider the discrete problem of VI:

$$
\left\{\begin{array}{l}
b\left(\bar{u}_{h}^{1}, v_{h}-\bar{u}_{h}^{1}\right) \geq\left(f\left(u_{0}\right)+\lambda u_{0}, v_{h}-\bar{u}_{h}^{1}\right), \quad v_{h} \in \mathbb{V}_{h}, \\
\bar{u}_{h}^{1} \leq r_{h} \psi, \quad v_{h} \leq r_{h} \psi .
\end{array}\right.
$$

Then as $\bar{u}_{h}^{1}$ is a solution to a discrete VI, it is also a subsolution, i.e.,

$$
\left\{\begin{array}{l}
b\left(\bar{u}_{h}^{1}, \varphi_{s}\right) \leq\left(f\left(u_{0}\right)+\lambda u_{0}, \varphi_{s}\right), \quad \forall \varphi_{s} \\
\bar{u}_{h}^{1} \leq r_{h} \psi
\end{array}\right.
$$


or

$$
\left\{\begin{array}{l}
b\left(\bar{u}_{h}^{1}, \varphi_{s}\right) \leq\left(f\left(u_{0}\right)+f\left(u_{0, h}\right)-f\left(u_{0, h}\right)+\lambda u_{0}-\lambda u_{0, h}+\lambda u_{0, h}, \varphi_{s}\right) \\
\bar{u}_{h}^{1} \leq r_{h} \psi
\end{array}\right.
$$

Then

$$
\left\{\begin{array}{l}
b\left(\bar{u}_{h}^{1}, \varphi_{s}\right) \leq\left(f\left(u_{0, h}\right)+\left\|f\left(u_{0}\right)-f\left(u_{0, h}\right)\right\|_{\infty}+\lambda\left\|u_{0}-u_{0, h}\right\|_{\infty}+\lambda u_{0, h}, \varphi_{s}\right) \\
\bar{u}_{h}^{1} \leq r_{h} \psi
\end{array}\right.
$$

Using the Lipschitz continuity of $f(\cdot)$, we have

$$
\left\{\begin{array}{l}
b\left(\bar{u}_{h}^{1}, \varphi_{s}\right) \leq\left(f\left(u_{0, h}\right)+\alpha\left\|u_{0}-u_{0, h}\right\|_{\infty}+\lambda\left\|u_{0}-u_{0, h}\right\|_{\infty}+\lambda u_{0, h}, \varphi_{s}\right) \\
\bar{u}_{h}^{1} \leq r_{h} \psi
\end{array}\right.
$$

On the other hand, due to [11]

$$
\left\|u_{0}-u_{0, h}\right\|_{\infty} \leq C h^{2}|\log h|
$$

Then

$$
\left\{\begin{array}{l}
b\left(\bar{u}_{h}^{1}, \varphi_{s}\right) \leq\left(f\left(u_{0, h}\right)+C h^{2}|\log h|+\lambda u_{0, h}, \varphi_{s}\right) \\
\bar{u}_{h}^{1} \leq r_{h} \psi
\end{array}\right.
$$

So, $\bar{u}_{h}^{1}$ is a discrete subsolution for the VI whose solution is $\bar{U}_{h}^{1}=\partial_{h}\left(f\left(u_{0, h}\right)+C h^{2}|\log h|\right.$, $\left.r_{h} \psi\right)$. Then $u_{h}^{1}=\partial_{h}\left(f\left(u_{0, h}\right), r_{h} \psi\right)$, and making use of Proposition 4 , we have

$$
\begin{aligned}
\left\|\bar{U}_{h}^{1}-u_{h}^{1}\right\|_{\infty} & \leq \frac{1}{\beta}\left\|f\left(u_{0, h}\right)+C h^{2}|\log h|-f\left(u_{0, h}\right)\right\|_{\infty} \\
& \leq C h^{2}|\log h| .
\end{aligned}
$$

Hence, making use of Theorem 4, we have

$$
\bar{u}_{h}^{1} \leq \bar{U}_{h}^{1} \leq u_{h}^{1}+C h^{2}|\log h|
$$

Putting

$$
\alpha_{h}^{1}=\bar{u}_{h}^{1}-C h^{2}|\log h|
$$

we get

$$
\alpha_{h}^{1} \leq u_{h}^{1}
$$

and

$$
\begin{aligned}
\left\|\alpha_{h}^{1}-u^{1}\right\|_{\infty} & =\left\|\bar{u}_{h}^{1}-C h^{2}|\log h|-u^{1}\right\|_{\infty} \\
& \leq\left\|\bar{u}_{h}^{1}-u^{1}\right\|_{\infty}+C h^{2}|\log h| .
\end{aligned}
$$


Using Lemma 3, we get

$$
\left\|\alpha_{h}^{1}-u^{1}\right\|_{\infty} \leq C h^{2}|\log h|^{2}+C h^{2}|\log h| .
$$

For $n+1$, let us now assume that

$$
\left\{\begin{array}{l}
\alpha_{h}^{n} \leq u_{h}^{n}, \\
\text { and } \\
\left\|\alpha_{h}^{n}-u^{n}\right\|_{\infty} \leq C h^{2}|\log h|^{2},
\end{array}\right.
$$

and we consider the discrete problem

$$
\left\{\begin{array}{l}
b\left(\bar{u}_{h}^{n+1}, v_{h}-\bar{u}_{h}^{n}\right) \geq\left(f\left(u^{n}\right)+\lambda u^{n}, v_{h}-\bar{u}_{h}^{n+1}\right), \quad v_{h} \in \mathbb{V}_{h} \\
\bar{u}_{h}^{n+1} \leq r_{h} \psi, \quad v_{h} \leq r_{h} \psi
\end{array}\right.
$$

Then

$$
\left\{\begin{array}{l}
b\left(\bar{u}_{h}^{n+1}, \varphi_{s}\right) \leq\left(f\left(u^{n}\right)+\lambda u^{n}, \varphi_{s}\right), \quad \forall \varphi_{s} \\
\bar{u}_{h}^{n+1} \leq r_{h} \psi
\end{array}\right.
$$

or

$$
\left\{\begin{array}{l}
b\left(\bar{u}_{h}^{n+1}, \varphi_{s}\right) \leq\left(f\left(u^{n}\right)+f\left(\bar{u}_{h}^{n}\right)-f\left(\bar{u}_{h}^{n}\right)+\lambda u^{n}-\lambda \bar{u}_{h}^{n}+\lambda \bar{u}_{h}^{n}, \varphi_{s}\right), \\
\bar{u}_{h}^{n+1} \leq r_{h} \psi
\end{array}\right.
$$

Then

$$
\left\{\begin{array}{l}
b\left(\bar{u}_{h}^{n+1}, \varphi_{s}\right) \leq\left(f\left(\bar{u}_{h}^{n}\right)+\left\|f\left(u^{n}\right)-f\left(\bar{u}_{h}^{n}\right)\right\|_{\infty}+\lambda\left\|u^{n}-\bar{u}_{h}^{n}\right\|_{\infty}+\lambda \bar{u}_{h}^{n}, \varphi_{s}\right) \\
\bar{u}_{h}^{n+1} \leq r_{h} \psi
\end{array}\right.
$$

Using the Lipschitz continuity of $f(\cdot)$, we have

$$
\left\{\begin{array}{l}
b\left(\bar{u}_{h}^{n+1}, \varphi_{s}\right) \leq\left(f\left(\bar{u}_{h}^{n}\right)+\alpha\left\|u^{n}-\bar{u}_{h}^{n}\right\|_{\infty}+\lambda\left\|u^{n}-\bar{u}_{h}^{n}\right\|_{\infty}+\lambda \bar{u}_{h}^{n}, \varphi_{s}\right), \\
\bar{u}_{h}^{n+1} \leq r_{h} \psi .
\end{array}\right.
$$

Using (4.2), we have

$$
\left\{\begin{array}{l}
b\left(\bar{u}_{h}^{n+1}, \varphi_{s}\right) \leq\left(f\left(\bar{u}_{h}^{n}\right)+C h^{2}|\log h|^{2}+\lambda \bar{u}_{h}^{n}, \varphi_{s}\right) \\
\bar{u}_{h}^{n+1} \leq r_{h} \psi
\end{array}\right.
$$

So, $\bar{u}_{h}^{n+1}$ is a discrete subsolution for the VI whose solution is $\bar{U}_{h}^{n+1}=\partial_{h}\left(f\left(\bar{u}_{h}^{n}\right)+\right.$ $\left.C h^{2}|\log h|^{2}, r_{h} \psi\right)$. Then $u_{h}^{n+1}=\partial_{h}\left(f\left(\bar{u}_{h}^{n}\right), r_{h} \psi\right)$, making use of Proposition 4, we have

$$
\begin{aligned}
\left\|\bar{u}_{h}^{n+1}-u_{h}^{n+1}\right\|_{\infty} & \leq \frac{1}{\beta}\left\|f\left(\bar{u}_{h}^{n}\right)+C h^{2}|\log h|^{2}-f\left(\bar{u}_{h}^{n}\right)\right\|_{\infty} \\
& \leq C h^{2}|\log h|^{2} .
\end{aligned}
$$


Hence, applying Theorem 4, we get

$$
\bar{u}_{h}^{n+1} \leq u_{h}^{n+1}+C h^{2}|\log h|^{2} .
$$

Putting

$$
\alpha_{h}^{n+1}=\bar{u}_{h}^{n+1}-C h^{2}|\log h|^{2},
$$

we get

$$
\alpha_{h}^{n+1} \leq u_{h}^{n+1}
$$

and

$$
\begin{aligned}
\left\|\alpha_{h}^{n+1}-u^{n+1}\right\|_{\infty} & =\left\|\bar{u}_{h}^{n+1}-C h^{2}|\log h|^{2}-u^{n+1}\right\|_{\infty} \\
& \leq\left\|\bar{u}_{h}^{n+1}-u^{n+1}\right\|_{\infty}+C h^{2}|\log h|^{2} .
\end{aligned}
$$

Using Lemma 3, we obtain

$$
\left\|\alpha_{h}^{n+1}-u^{n+1}\right\|_{\infty} \leq C h^{2}|\log h|^{2},
$$

which completes the proof.

4.1.2 A continuous sequence

We define the following continuous sequence $\left\{\bar{u}_{(h)}^{n}\right\}_{n \geq 1}$, where $\bar{u}_{(h)}^{n}$ is a solution to the following continuous problem of variational inequalities (VI):

$$
\left\{\begin{array}{l}
b\left(\bar{u}_{(h)}^{n}, v-\bar{u}_{(h)}^{n}\right) \geq\left(f\left(u_{h}^{n}\right)+\lambda \cdot u_{h}^{n}, v-\bar{u}_{(h)}^{n}\right), \quad v \in H_{0}^{1}(\Omega) \\
\bar{u}_{(h)}^{n} \leq \psi, \quad v \leq \psi
\end{array}\right.
$$

where $u_{h}^{n}$ is the solution of discrete problem (3.4).

Lemma 4 (cf. [13]) There exists a constant $C$ independent of $h, k$, and $n$ such that

$$
\left\|\bar{u}_{(h)}^{n}-u_{h}^{n}\right\|_{\infty} \leq C h^{2}|\log h|^{2},
$$

where the constant $C$ is independent of $h, n$, and $\Delta t$.

Proposition 6 There exists a sequence of continuous subsolutions $\left\{\beta_{(h)}^{n}\right\}_{n \geq 1}$ such that

$$
\left\{\begin{array}{l}
\beta_{(h)}^{n} \leq u^{n} \\
\text { and } \\
\left\|\beta_{(h)}^{n}-u_{h}^{n}\right\|_{\infty} \leq C h^{2}|\log h|^{2},
\end{array}\right.
$$

where the constant $C$ is independent of $h, \Delta t$, and $n$. 
Proof For $n=1$, we consider the continuous problem of VI

$$
\left\{\begin{array}{l}
b\left(\bar{u}_{(h)}^{1}, v-\bar{u}_{(h)}^{1}\right) \geq\left(f\left(u_{0, h}\right)+\lambda u_{0, h}, v-\bar{u}_{(h)}^{1}\right), \quad v \in H_{0}^{1}(\Omega) \\
\bar{u}_{(h)}^{1} \leq \psi, \quad v \leq \psi
\end{array}\right.
$$

Then, as $\bar{u}_{(h)}^{1}$ is a solution to a continuous VI, it is also a subsolution, i.e.,

$$
\left\{\begin{array}{l}
b\left(\bar{u}_{(h)}^{1}, v\right) \leq\left(f\left(u_{0, h}\right)+\lambda u_{0, h}, v\right) \\
\bar{u}_{(h)}^{1} \leq \psi
\end{array}\right.
$$

or

$$
\left\{\begin{array}{l}
b\left(\bar{u}_{(h)}^{1}, v\right) \leq\left(f\left(u_{0, h}\right)+f\left(u_{0}\right)-f\left(u_{0}\right)+\lambda u_{0, h}-\lambda u_{0}+\lambda u_{0}, v\right) \\
\bar{u}_{(h)}^{1} \leq \psi
\end{array}\right.
$$

Then

$$
\left\{\begin{array}{l}
b\left(\bar{u}_{(h)}^{1}, v\right) \leq\left(f\left(u_{0}\right)+\left\|f\left(u_{0}\right)-f\left(u_{0, h}\right)\right\|_{\infty}+\lambda\left\|u_{0}-u_{0, h}\right\|_{\infty}+\lambda u_{0}, v\right) \\
\bar{u}_{(h)}^{1} \leq \psi
\end{array}\right.
$$

Using the Lipschitz continuity of $f(\cdot)$, we have

$$
\left\{\begin{array}{l}
b\left(\bar{u}_{(h)}^{1}, v\right) \leq\left(f\left(u_{0}\right)+\alpha\left\|u_{0}-u_{0, h}\right\|_{\infty}+\lambda\left\|u_{0}-u_{0, h}\right\|_{\infty}+\lambda u_{0}, v\right) \\
\bar{u}_{(h)}^{1} \leq \psi
\end{array}\right.
$$

On the other hand, due to [11]

$$
\left\|u_{0}-u_{0, h}\right\|_{\infty} \leq C h^{2}|\log h|
$$

Then

$$
\left\{\begin{array}{l}
b\left(\bar{u}_{(h)}^{1}, v\right) \leq\left(f\left(u_{0}\right)+C h^{2}|\log h|+\lambda u_{0}, v\right) \\
\bar{u}_{(h)}^{1} \leq \psi
\end{array}\right.
$$

So, $\bar{u}_{(h)}^{1}$ is a continuous subsolution for the VI whose solution is $\bar{U}_{(h)}^{1}=\partial\left(f\left(u_{0}\right)+\right.$ $\left.C h^{2}|\log h|, \psi\right)$. Then $u^{1}=\partial\left(f\left(u_{0}\right), \psi\right)$, and making use of Proposition 2, we have

$$
\begin{aligned}
\left\|\bar{U}_{(h)}^{1}-u^{1}\right\| & \leq \frac{1}{\beta}\left\|f\left(u_{0}\right)+C h^{2}|\log h|-f\left(u_{0}\right)\right\|_{\infty} \\
& \leq C h^{2}|\log h| .
\end{aligned}
$$

Hence, making use of Theorem 2, we have

$$
\bar{u}_{(h)}^{1} \leq \bar{U}_{(h)}^{1} \leq u^{1}+C h^{2}|\log h|^{2}
$$


Putting

$$
\beta_{(h)}^{1}=\bar{u}_{(h)}^{1}-C h^{2}|\log h|^{2}
$$

we get

$$
\beta_{(h)}^{1} \leq u^{1}
$$

and

$$
\begin{aligned}
\left\|\beta_{(h)}^{1}-u_{h}^{1}\right\|_{\infty} & =\left\|\bar{u}_{(h)}^{1}-C h^{2}|\log h|^{2}-u_{h}^{1}\right\|_{\infty} \\
& \leq\left\|\bar{u}_{(h)}^{1}-u_{h}^{1}\right\|_{\infty}+C h^{2}|\log h|^{2} .
\end{aligned}
$$

Using Lemma 4, we obtain

$$
\left\|\beta_{(h)}^{1}-u_{h}^{1}\right\|_{\infty} \leq C h^{2}|\log h|^{2} .
$$

For $n+1$, let us now assume that

$$
\left\{\begin{array}{l}
\beta_{(h)}^{n} \leq u^{n} \\
\text { and } \\
\left\|\beta_{(h)}^{n}-u_{h}^{n}\right\|_{\infty} \leq C h^{2}|\log h|^{2}
\end{array}\right.
$$

and consider the continuous problem

$$
\left\{\begin{array}{l}
b\left(\bar{u}_{(h)}^{n+1}, v-\bar{u}_{(h)}^{n+1}\right) \geq\left(f\left(u_{h}^{n}\right)+\lambda u_{h}^{n}, v-\bar{u}_{(h)}^{n+1}\right), \quad v \in H_{0}^{1}(\Omega), \\
\bar{u}_{(h)}^{n+1} \leq \psi, \quad v \leq \psi
\end{array}\right.
$$

Then

$$
\left\{\begin{array}{l}
b\left(\bar{u}_{(h)}^{n+1}, v\right) \leq\left(f\left(u_{h}^{n}\right)+\lambda u_{h}^{n}, v\right), \quad v \in H_{0}^{1}(\Omega), \\
\bar{u}_{(h)}^{n+1} \leq \psi
\end{array}\right.
$$

or

$$
\left\{\begin{array}{l}
b\left(\bar{u}_{(h)}^{n+1}, v\right) \leq\left(f\left(u_{h}^{n}\right)+f\left(\bar{u}_{(h)}^{n}\right)-f\left(\bar{u}_{(h)}^{n}\right)+\lambda \bar{u}_{(h)}^{n}-\lambda \bar{u}_{(h)}^{n}+\lambda u_{h}^{n}, v\right), \\
\bar{u}_{(h)}^{n+1} \leq \psi
\end{array}\right.
$$

Then

$$
\left\{\begin{array}{l}
b\left(\bar{u}_{(h)}^{n+1}, v\right) \leq\left(f\left(\bar{u}_{(h)}^{n}\right)+\left\|f\left(u_{h}^{n}\right)-f\left(\bar{u}_{(h)}^{n}\right)\right\|_{\infty}+\lambda\left\|\bar{u}_{(h)}^{n}-u_{h}^{n}\right\|_{\infty}+\lambda \bar{u}_{(h)}^{n}, v\right), \\
\bar{u}_{(h)}^{n+1} \leq \psi
\end{array}\right.
$$

Using the Lipschitz continuity of $f(\cdot)$, we have

$$
\left\{\begin{array}{l}
b\left(\bar{u}_{(h)}^{n+1}, v\right) \leq\left(f\left(\bar{u}_{(h)}^{n}\right)+\alpha\left\|\bar{u}_{(h)}^{n}-u_{h}^{n}\right\|_{\infty}+\lambda\left\|\bar{u}_{(h)}^{n}-u_{h}^{n}\right\|_{\infty}+\lambda \bar{u}_{(h)}^{n}, v\right), \\
\bar{u}_{(h)}^{n+1} \leq \psi
\end{array}\right.
$$


Using (4.4), we have

$$
\left\{\begin{array}{l}
b\left(\bar{u}_{(h)}^{n+1}, v\right) \leq\left(f\left(\bar{u}_{(h)}^{n}\right)+C h^{2}|\log h|^{2}+\lambda \bar{u}_{(h)}^{n}, v\right), \\
\bar{u}_{(h)}^{n+1} \leq \psi
\end{array}\right.
$$

So, $\bar{u}_{(h)}^{n+1}$ is a continuous subsolution for the VI whose solution is $\bar{U}_{(h)}^{n+1}=\partial\left(f\left(\bar{u}_{(h)}^{n}\right)+\right.$ $\left.C h^{2}|\log h|^{2}, \psi\right)$. Then $u^{n+1}=\partial\left(f\left(\bar{u}_{(h)}^{n}\right), \psi\right)$, and making use of Proposition 2, we have

$$
\begin{aligned}
\bar{u}_{(h)}^{n+1}-u^{n+1} & \leq C\left(\left\|f\left(\bar{u}_{(h)}^{n}\right)+C h^{2}|\log h|^{2}-f\left(\bar{u}_{(h)}^{n}\right)\right\|_{\infty}\right) \\
& \leq C h^{2}|\log h|^{2}
\end{aligned}
$$

and, making use of Theorem 2, we obtain

$$
\bar{u}_{(h)}^{n+1} \leq u^{n+1}+C h^{2}|\log h|^{2} .
$$

Now, taking

$$
\beta_{(h)}^{n+1}=\bar{u}_{(h)}^{n+1}-C h^{2}|\log h|^{2},
$$

we have

$$
\beta_{(h)}^{n+1} \leq u^{n+1}
$$

and

$$
\begin{aligned}
\left\|\beta_{(h)}^{n+1}-u_{h}^{n+1}\right\|_{\infty} & =\left\|\bar{u}_{(h)}^{n+1}-C h^{2}|\log h|^{2}-u_{h}^{n+1}\right\|_{\infty} \\
& \leq\left\|\bar{u}_{(h)}^{n+1}-u_{h}^{n+1}\right\|_{\infty}+C h^{2}|\log h|^{2} .
\end{aligned}
$$

Using Lemma 4, we obtain

$$
\left\|\beta_{(h)}^{n+1}-u_{h}^{n+1}\right\|_{\infty} \leq C h^{2}|\log h|^{2},
$$

which completes the proof.

\section{$4.2 L^{\infty}$-Error estimate}

Now, guided by Propositions 5 and 6, we are in a position to prove the following.

Theorem 5 Under the conditions of Propositions 5 and 6, we have

$$
\left\|u^{n}-u_{h}^{n}\right\|_{\infty} \leq C h^{2}|\log h|^{2}
$$

where the constant $C$ is independent of $h, \Delta t$, and $n$.

Proof Using (4.3), we have

$$
\begin{aligned}
u^{n} & \leq \alpha_{h}^{n}+C h^{2}|\log h|^{2} \\
& \leq u_{h}^{n}+C h^{2}|\log h|^{2},
\end{aligned}
$$


thus

$$
u^{n}-u_{h}^{n} \leq C h^{2}|\log h|^{2},
$$

and using (4.6), we have

$$
\begin{aligned}
u_{h}^{n} & \leq \beta_{(h)}^{n}+C h^{2}|\log h|^{2} \\
& \leq u^{n}+C h^{2}|\log h|^{2} .
\end{aligned}
$$

Thus, we get

$$
u_{h}^{n}-u^{n} \leq C h^{2}|\log h|^{2} .
$$

Therefore

$$
\left\|u^{n}-u_{h}^{n}\right\|_{\infty} \leq C h^{2}|\log h|^{2}
$$

which completes the proof.

Corollary 1 In (4.7), passing to the limit, as $n \rightarrow+\infty$, we get

$$
\left\|u^{\infty}-u_{h}^{\infty}\right\|_{\infty} \leq C h^{2}|\log h|^{2} .
$$

\section{3 $L^{\infty}$-Asymptotic behavior}

Now we estimate the order of the difference between $u_{h}(T, \cdot)$, the discrete solution calculated at the moment $T=n \Delta t$, and $u^{\infty}$, the solution of problem (2.13).

Theorem 6 (The main result) Under the conditions of Proposition 3 and Corollary 1, the following inequality holds:

$$
\left\|u_{h}(T, \cdot)-u^{\infty}(\cdot)\right\|_{\infty} \leq C\left(h^{2}|\log h|^{2}+\left(\frac{\alpha \Delta t+1}{\beta \Delta t+1}\right)^{N}\right) .
$$

Proof We have

$$
u_{h}^{n}(t, \cdot)=u_{h}(t, \cdot) \quad \text { for all } t \in((n-1) \Delta t, n \Delta t),
$$

thus

$$
\begin{aligned}
\left\|u_{h}(T, \cdot)-u^{\infty}(\cdot)\right\|_{\infty} & =\left\|u_{h}^{N}(\cdot)-u^{\infty}(\cdot)\right\|_{\infty} \\
& \leq\left\|u_{h}^{N}-u_{h}^{\infty}\right\|_{\infty}+\left\|u_{h}^{\infty}-u^{\infty}\right\|_{\infty} .
\end{aligned}
$$

Indeed, applying the previous results of Proposition 3 and Corollary 1, we get

$$
\left\|u_{h}(T, \cdot)-u^{\infty}(\cdot)\right\|_{\infty} \leq\left(\frac{\alpha \Delta t+1}{\beta \Delta t+1}\right)^{N}\left\|u_{h}^{0}-u_{h}^{\infty}\right\|_{\infty}+C h^{2}|\log h|^{2} .
$$


Then the following result can be deduced:

$$
\left\|u_{h}(T, x)-u^{\infty}(x)\right\|_{\infty} \leq C\left(h^{2}|\log h|^{2}+\left(\frac{\alpha \Delta t+1}{\beta \Delta t+1}\right)^{N}\right)
$$

which completes the proof.

\section{Acknowledgements}

The authors would like to thank the anonymous referees and the handling editor for their careful reading and for relevant remarks/suggestions which helped them to improve the paper.

\section{Funding}

Not applicable.

\section{Availability of data and materials}

Not applicable.

\section{Ethics approval and consent to participate}

Not applicable.

\section{Competing interests}

The authors declare that there is no conflict of interests regarding the publication of this manuscript. The authors declare that they have no competing interests.

\section{Consent for publication}

Not applicable.

Authors' contributions

The authors contributed equally in this article. They have all read and approved the final manuscript.

\section{Author details}

'Department of Mathematics, College of Sciences and Arts, Al-Ras Qassim University, Buraydah, Kingdom of Saudi Arabia. ${ }^{2}$ Laboratory of Fundamental and Applied Mathematics of Oran (LMFAO), University of Oran 1, Ahmed Benbella, Algeria.

${ }^{3}$ Tamanghesset University Center, Sersouf, Algeria. ${ }^{4}$ LANOS Laboratory, Department of Mathematics, Faculty of Sciences, Badji Mokhtar University, Annaba, Algeria.

\section{Publisher's Note}

Springer Nature remains neutral with regard to jurisdictional claims in published maps and institutional affiliations.

Received: 3 January 2020 Accepted: 18 March 2020 Published online: 24 March 2020

\section{References}

1. Achdou, A., Hecht, F., Pommier, D.: A posteriori error estimates for parabolic variational inequalities. J. Sci. Comput. Math. Appl. 37(3), 336-366 (2008)

2. Bencheikh Le Hocine, M.A., Boulaaras, S., Haiuor, M.: An optimal $L^{\infty}$-error estimate for an approximation of a parabolic variational inequality. Numer. Funct. Anal. Optim. 37(1), 1-18 (2016)

3. Benssoussan, A., Lions, J.L.: Applications des inéquations variationnelles en contrôle stochastique. Dunod, Paris (1978)

4. Berger, A., Falk, R.: An error estimate for the truncation method for the solution of parabolic obstacle variational inequalities. Math. Comput. 31(139), 619-628 (1977)

5. Boulaaras, S.: An optimal error estimate of finite element method for parabolic quasi-variational inequalities with nonlinear source terms. Asymptot. Anal. 100(3-4), 193-208 (2016)

6. Boulaaras, S.: Some new properties of asynchronous algorithms of theta scheme combined with finite elements methods for an evolutionary implicit two-sided obstacle problem. Math. Methods Appl. Sci. 40, 7231-7239 (2017)

7. Boulaaras, S., Haiour, M.: $L^{\infty}$-Asymptotic behavior for a finite element approximation in parabolic quasi-variational inequalities related to impulse control problem. Appl. Math. Comput. 217, 6443-6450 (2011)

8. Boulaaras, S., Haiour, M.: A new approach to asymptotic behavior for a finite element approximation in parabolic variational inequalities. ISRN Math. Anal. 2011, Article ID 70367 (2011)

9. Boulaaras, S., Haiour, M.: The finite element approximation of evolutionary Hamilton-Jacobi-Bellman equations with nonlinear source terms. Indag. Math. 24(1), 161-173 (2013)

10. Boulaaras, S., Haiour, M.: A new proof for the existence and uniqueness of the discrete evolutionary HJB equations. Appl. Math. Comput. 262(14), 42-55 (2015)

11. Boulaaras, S., Touati Brahim, M., Bouznada, S.: A posteriori error estimates for the generalized Schwarz method of a class of advection-diffusion equation with mixed boundary condition. Math. Methods Appl. Sci. 41(14), 5493-5505 (2018)

12. Boulaaras, S., Touati Brahim, M.-S., Bouzenada, S., Zarai, A.: An a posteriori error estimates of the generalized Shwarz method for advection-diffusion equation. Acta Math. Sci. 38(4), 1227-1244 (2018)

13. Boulbrachene, $M$.: Optimal $L^{\infty}$-error estimate for variational inequalities with nonlinear source terms. Appl. Math. Lett. 15(8), 1013-1017 (2002) 
14. Ciarlet, P.G., Raviart, P.A.: Maximum principle and uniform convergence for the finite element method. Comput. Math. Appl. 2(1), 1-20 (1973)

15. Cortey-Dumont, $P$.: On finite element approximation in the $L^{\infty}$-norm of parabolic obstacle variational inequalities and quasi-variational inequalities, Rapport interne $\mathrm{n}^{\circ}$ 112, CMA Palaiseau, France

16. Duvaut, G., Lions, J.L.: Les inéquations en mechanique et en physique. Dunod, Paris (1972)

17. Fetter, A.: $L^{\infty}$-Estimate for an approximation of a parabolic variational inequality. Numer. Math. 50(5), 557-565 (1987)

18. Glowinski, R., Lions, J.L., Tremolieres, R.: Numerical Analysis of Variational Inequalities. North-Holland, Amsterdam (1981)

19. Han, W., Sofonea, M.: Evolutionary variational inequalities arising in viscoelastic contact problems. SIAM J. Numer. Anal. 38(2), 556-579 (2000)

20. Johnson, C.: A convergence estimate for an approximation of a parabolic variational inequality. SIAM J. Numer. Anal. 13(4), 599-606 (1976)

21. Kinderlehrer, D., Stampacchia, G.: An Introduction to Variational Inequalities and Their Application. Academic Press, New York (1980)

22. Kornhuber, R.: A posteriori error estimates for elliptic parabolic variational inequalities. Comput. Math. Appl. 31(8), 49-60 (1996)

23. Vuik, C.: An $L^{2}$-error estimate for an approximation of the solution of a parabolic variational inequality. Numer. Math 57(1), 453-471 (1990)

Submit your manuscript to a SpringerOpen ${ }^{\odot}$ journal and benefit from:

- Convenient online submission

- Rigorous peer review

- Open access: articles freely available online

- High visibility within the field

- Retaining the copyright to your article

Submit your next manuscript at $\boldsymbol{~ s p r i n g e r o p e n . c o m ~}$ 